\title{
A Simulation Study on Channel Estimation for Cooperative Communication System in Sand-dust Storm Environment
}

\author{
Xuehong Sun ${ }^{1,2}$, Yu Cao ${ }^{1}$, Jin Che ${ }^{1}$ \\ ${ }^{1}$ School of Physics and Electrical Information Engineering, Ningxia University, YinChuan, China \\ ${ }^{2}$ School of Information and Communication Engineering, Beijing University of Posts and Telecommunications, Beijing, China \\ Email: nxsunxh@gmail.com
}

Received May, 2013

\begin{abstract}
There are many factors that influence the propagation of electromagnetic wave in the sand-dust storm environment, the scattering effect of dust particle is one of the major factors, so this paper focuses on the dust particles scattering function. The scattering of dust particles inevitably brings the multipath transmission of the signal, multipath propagation will bring the multipath fading of the signal. In this paper, we first investigate the use of AF and DF modes in a sand-dust storm environment. Secondly, we present a low-order modulation method should be used in cooperative communication system. Lastly, we evaluate the system performance for both of the moving nodes and power allocation. Experimental results validate the conclusion of theoretical derivation: the multipath fading is one of the main factors that affect the quality of signal transmission. Cooperative communication technology has good anti-fading ability, which can guarantee the signal transmission timely and correctly.
\end{abstract}

Keywords: Cooperative Communication; Sand-dust Storm; Particle Scattering; Multipath Fading; OFDM

\section{Introduction}

During the wireless communications, the main factors that influenced the quality of wireless communication are the complexity of the transmission environment and multipath fading of wireless signals. The transmission environment in the sand-dust storm is more complex than that in the urban environment. There are many factors that can influence the transmission of electromagnetic wave, firstly, the loss of signal transmission in the sandstorm channels; secondly, the attenuation that caused by the water content and visibility of the sandstorm; thirdly, the polarization effect of dust particles influence on electromagnetic waves. Last, the scattering effect of dust particles.

This paper discusses cooperative communication system in sand-dust storm environment. It does research on sand-dust storm communication system, for the project of Ningxia University The research on access to information technology cooperation in desert hinterland dust monitoring.

It is a project for information acquisition of desert and sand-dust storm. The implementation environment of the project is in desert hinterland, about 25 kilometers. It uses the sensors carried by robots to get a variety of data in desert. The data is transmitted to remote information center by wireless communication network. In desert hinterland, each node can be real-time mobile robots.
With the moving of the nodes, the communication network is also changing. When a node is moving, a node must leave or enter the coverage situation of other communication nodes, information transmission is required in the cooperation of other nodes to complete. In addition, the nodes may move into the position of the sand dunes, which will cause that the information cannot reach the destination node by direct passing way, this also need other nodes together to complete the data transmission.

When the electromagnetic wave is transmitting in the sand-dust storm environment, it will be affected by dust particles. Dust particles will cause the scattering effect. The scattering of dust particles inevitably brings the multipath transmission of the signal, multipath propagation will bring the multipath fading of the signal.

In this paper, we do the study of cooperative communication mode and dust environment, and look for a suitable cooperative communication system, which can work in sand-dust storm environment.

\section{Theory Explanation}

\subsection{Cooperative Communication Model}

\subsubsection{AF model and its performance}

Amplify-and-forward (AF) is one of the most simple way in cooperative communication. It is same as Decodeand-forward (DF) model in the first time slot. However, 
in the second time slot, it amplifies the signals received by relay and sent it directly. And use MRC (Maximum Ratio Combining) to do signal processing for the first and second time slot[2].

$$
y=a_{1} y_{s, d}+a_{2} y_{r, d}
$$

where

$$
a_{1}=\frac{\sqrt{P_{s}} h_{s, d}^{*}}{\sigma_{0}^{2}}, \quad a_{2}=\frac{\sqrt{\frac{P_{s} P_{r}}{\left|h_{s, r}\right|^{2} P_{s}+\sigma_{0}^{2}}} h_{s, r}^{*} h_{r, d}^{*}}{\left(\frac{P_{r}\left|h_{r, d}\right|^{2}}{\left|h_{s, r}\right|^{2} P_{s}+\sigma_{0}^{2}}+1\right) \sigma_{0}^{2}}
$$

marks conjugate transpose, the instantaneous SNR is $\gamma=\gamma_{1}+\gamma_{2}$ [1], $\gamma_{1}$ is the SNR of the source node to destination node direct passing channel, $\gamma_{2}$ is the SNR of relay node to destination node.

$$
\begin{gathered}
\gamma_{1}=\frac{P_{s}}{\sigma_{0}^{2}}\left|h_{s, d}\right|^{2} \\
\gamma_{2}=\frac{\frac{P_{s}}{\sigma_{0}^{2}}\left|h_{s, d}\right|^{2} \cdot \frac{P_{r}}{\sigma_{0}^{2}}\left|h_{r, d}\right|^{2}}{\frac{P_{s}}{\sigma_{0}^{2}}\left|h_{s, d}\right|^{2} \cdot \frac{P_{r}}{\sigma_{0}^{2}}\left|h_{r, d}\right|^{2}+1}
\end{gathered}
$$

According to the reference [2], instantaneous $\gamma_{2}$ is:

$$
\hat{\gamma}_{2}=\frac{\frac{P_{s}}{\sigma_{0}^{2}}\left|h_{s, d}\right|^{2} \cdot \frac{P_{r}}{\sigma_{0}^{2}}\left|h_{r, d}\right|^{2}}{\frac{P_{s}}{\sigma_{0}^{2}}\left|h_{s, d}\right|^{2} \cdot \frac{P_{r}}{\sigma_{0}^{2}}\left|h_{r, d}\right|^{2}}
$$

And use MQAM (Multiple Quadrature Amplitude Modulation) to do the analysis of the AF mode.

MQAM is a kind of carrier wave control mode that widely used in the capacity and the large capacity digital microwave communication system. It can improve the spectrum efficiency of communication system. At present, in large capacity digital microwave communication system, such as SDH (Synchronous Digital Hierarchy) digital microwave and LMDS, there are widely used in 64 QAM and 128 QAM, which are MQAM modulation mode.

According to the reference [3], the symbol error rate (SER):

$$
\begin{aligned}
P_{M Q A M}^{h_{s, d} h_{s, r} h_{r, d}} \approx & 4 K Q\left(\sqrt{b_{M Q A M}\left(\gamma_{1}+\hat{\gamma}_{2}\right)}\right) \\
& -4 K^{2} Q^{2}\left(\sqrt{b_{M Q A M}\left(\gamma_{1}+\hat{\gamma}_{2}\right)}\right)
\end{aligned}
$$

where

$$
K=1-\frac{1}{\sqrt{M}}, \quad b_{M Q A M}=\frac{3}{M-1},
$$

$$
\begin{aligned}
Q \text { is } \quad Q(z) & =\frac{1}{\pi} \int_{0}^{\pi / 2} \exp \left(-\frac{z^{2}}{2 \sin ^{2} \theta}\right) d \theta \\
Q^{2}(z) & =\frac{1}{\pi} \int_{0}^{\pi / 4} \exp \left(-\frac{z^{2}}{2 \sin ^{2} \theta}\right) d \theta
\end{aligned}
$$

Average SER is:

$P_{M Q A M}^{A F}$

$=\frac{4 K}{\pi} \int_{0}^{\pi / 2} \frac{2 \sin ^{2} \theta \sigma_{0}^{2}}{b_{M Q A M} P_{s} \Omega_{s, d}} \cdot \frac{2 \sin ^{2} \theta \sigma_{0}^{2}\left(P_{s} \Omega_{s, r}+P_{r} \Omega_{r, d}\right)}{b_{M Q A M} P_{s} P_{r} \Omega_{s, r} \Omega_{r, d}} d \theta$

$-\frac{4 K^{2}}{\pi} \int_{0}^{\pi / 4} \frac{2 \sin ^{2} \theta \sigma_{0}^{2}}{b_{M Q A M} P_{s} \Omega_{s, d}} \cdot \frac{2 \sin ^{2} \theta \sigma_{0}^{2}\left(P_{s} \Omega_{s, r}+P_{r} \Omega_{r, d}\right)}{b_{M Q A M} P_{s} P_{r} \Omega_{s, r} \Omega_{r, d}}$

$=\frac{A \sigma_{0}^{4}\left(P_{s} \Omega_{s, r}+P_{r} \Omega_{r, d}\right)}{\left(b_{\text {MQAM }} / 2\right)^{2} P_{s}^{2} P_{r} \Omega_{s, r} \Omega_{r, d}}$

$=\frac{A \sigma_{0}^{4}}{\left(b_{M Q A M} / 2\right)^{2}} \cdot \frac{1}{P_{s} \Omega_{s, d}}\left(\frac{1}{P_{s} \Omega_{s, r}}+\frac{1}{P_{r} \Omega_{r, d}}\right)$

where

$A=\frac{4 K}{\pi} \int_{0}^{\pi / 2} \sin ^{4} \theta d \theta-\frac{4 K^{2}}{\pi} \int_{0}^{\pi / 4} \sin ^{4} \theta d \theta=\frac{3(M-1)}{8 M}+\frac{K^{2}}{\pi}$

The Figure 1 is the performance curve of 16 QAM and 4 QAM modulation system in AF mode, where $\Omega_{s, d}=$ $\Omega_{s, r}=\Omega_{r, d}=1, \quad \sigma_{0}^{2}=1, K=3 / 4, \quad b_{16 Q A M}=1 / 5$. Curve shows that the performance of $4 \mathrm{QAM}$ is better than 16QAM. In low SER, the difference of two methods is not bit. With the increase of SNR, the good performance of 4QAM gradually reflected. Therefore, the low-order modulation method should be used in AF mode.

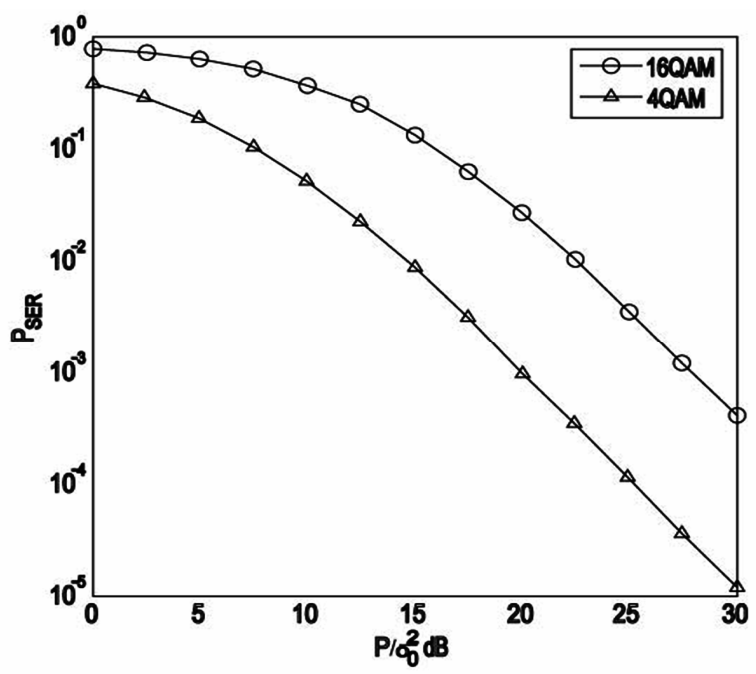

Figure 1. The performance curve of 16 QAM and 4 QAM modulation system in AF mode. 


\subsubsection{DF Model and Its Performance}

Decode-and-forward(DF) is another way in cooperative communication. In DF model, the relay receives the signal and does demodulation and decoding. Then, the verdict will be done; the relay will do recoding for the data and send it out again. The study of DF model also can use MRC.

$$
y=a_{1} y_{s, d}+a_{2} y_{r, d}
$$

where

$$
a_{1}=\sqrt{P_{s}} h_{s, d}^{*} / \sigma_{0}^{2}, a_{2}=\sqrt{P_{r}} h_{r, d}^{*} / \sigma_{0}^{2}
$$

The SNR of the receiver is:

$$
\gamma=\frac{P_{s}\left|h_{s, d}\right|^{2}+\tilde{P}_{r}\left|h_{r, d}\right|^{2}}{\sigma_{0}^{2}}
$$

In MQAM modulation, the approximate SER of the system is:

$$
\begin{aligned}
P_{M Q A M}^{D F} \approx & F_{2}\left(\frac{b_{M Q A M} P_{s} \Omega_{s, d}}{2 \sigma_{0}^{2} \sin ^{2} \theta}\right) F_{2}\left(\frac{b_{M Q A M} P_{s} \Omega_{s, r}}{2 \sigma_{0}^{2} \sin ^{2} \theta}\right) \\
& +F_{2}\left(\frac{b_{M Q A M}^{2} P_{s} P_{r} \Omega_{s, d} \Omega_{r, d}}{4 \sigma_{0}^{4} \sin ^{4} \theta}\right) \\
& \times\left[1-F_{2}\left(1+\frac{b_{M Q A M} P_{s} \Omega_{s, r}}{2 \sigma_{0}^{2} \sin ^{2} \theta}\right)\right] \\
= & F_{2}\left(\frac{b_{M Q A M} P_{s} \Omega_{s, d}}{2 \sigma_{0}^{2} \sin ^{2} \theta}\right) F_{2}\left(\frac{b_{M Q A M} P_{s} \Omega_{s, r}}{2 \sigma_{0}^{2} \sin ^{2} \theta}\right) \\
& +F_{2}\left(\frac{b_{M Q A M}^{2} P_{s} P_{r} \Omega_{s, d} \Omega_{r, d}}{4 \sigma_{0}^{4} \sin ^{4} \theta}\right) \\
= & \frac{4 B^{2} \sigma_{0}^{4}}{b_{M Q A M}^{2} P_{s}^{2} \Omega_{s, d} \Omega_{s, r}}+\frac{4 C \sigma_{0}^{4}}{b_{M Q A M}^{2} P_{s} P_{r} \Omega_{s, d} \Omega_{r, d}}
\end{aligned}
$$

where

$$
\begin{aligned}
B & =\frac{4 K}{\pi \sqrt{M}} \int_{0}^{\pi / 2} \sin ^{2} \theta d \theta+\frac{4 K^{2}}{\pi} \int_{\pi / 4}^{\pi / 2} \sin ^{2} \theta d \theta \\
& =\frac{M-1}{2 M}+\frac{K^{2}}{\pi} \\
C & =\frac{4 K}{\pi \sqrt{M}} \int_{0}^{\pi / 2} \sin ^{4} \theta d \theta+\frac{4 K^{2}}{\pi} \int_{\pi / 4}^{\pi / 2} \sin ^{4} \theta d \theta \\
& =\frac{3(M-1)}{8 M}+\frac{K^{2}}{\pi}
\end{aligned}
$$

The Figure 2 is the performance curve of 16 QAM and 4 QAM modulation systems in DF mode. It is the same as AF model.

Where $\Omega_{s, d}=\Omega_{s, r}=\Omega_{r, d}=1, \quad \sigma_{0}^{2}=1, K=3 / 4$, $b_{16 Q A M}=1 / 5$.

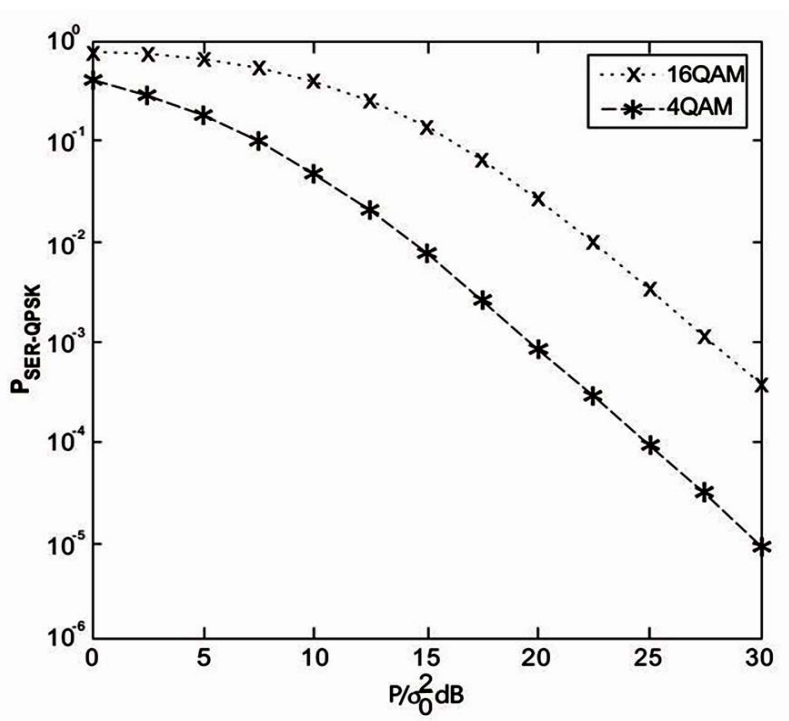

Figure 2. The performance curve of 16 QAM and 4 QAM modulation system in DF mode.

With the increase of SNR, the good performance of 4 QAM gradually reflected. Therefore, the low-order modulation method should be used in DF mode, as well.

In the research of AF and DF system performance, it can draw the conclusion: in the case of high SNR, the low-order modulation mode should be used to keep the SER of the system in the low condition.

\subsection{Influence of Propagation}

\subsubsection{Polarization Influence on Electromagnetic Wave}

From the references [4] and [5], the scattering and absorption of dust particles can cause the attenuation of electromagnetic wave. Because the shapes of the dust particles are irregular, when the electromagnetic wave spreads in the sand-dust storm, differential attenuation and differential phase shift is on the different directions. Dust particles can be seen as a spheroid, the ratio of its axis is:

$$
a: b: c=1: 0.75:(0.75)^{2}
$$

According to the equivalent medium theory, dust particles can be equivalent to homogeneous medium. It is assumed that the radius of all particle are the same, according to the reference [6], we can get the following formula:

$$
\begin{gathered}
\alpha=-\frac{0.6287 f}{V_{b}} a_{e} \operatorname{Im}\left[\frac{\varepsilon_{m}^{*}-1}{\varepsilon_{m}^{*}+2}\right] \\
\beta=\frac{4.15 f}{V_{b}} a_{e} \operatorname{Re}\left[\frac{\varepsilon_{m}^{*}-1}{\varepsilon_{m}^{*}+2}\right]
\end{gathered}
$$

where $\varepsilon_{m}^{*}$ is the dielectric constant of dust particles, $a_{e}$ 
is the equivalent radius of dust particles, $V_{b}$ is the visibility of sand-dust storm, $\alpha$ is the attenuation coefficient, $\beta$ is the phase shift coefficient.

$$
a_{e}=\frac{\int_{a \min }^{a \max } a^{3} p(a) d a}{\int_{a \text { min }}^{a \text { max }} a^{2} p(a) d a}
$$

In sand-dust storm environment, the differential attenuation coefficient and the differential phase shift [7] of electromagnetic wave are:

$$
\begin{gathered}
\Delta \alpha=\left|a_{h}-a_{v}\right|=2.099 \times 10^{2} \frac{f}{V_{b}} a_{e} \\
\left|\frac{1}{2}\left(L_{1}^{\prime \prime}+L_{2}^{\prime \prime}\right)-L_{3}^{\prime \prime}\right| \cdot\left(\frac{\bar{b}}{a}\right) \cdot \sin ^{2} \phi \\
\Delta \beta=\left|\beta_{h}-\beta_{v}\right|=1.3848 \times 10^{3} \frac{f}{V_{b}} a_{e} \\
\left|\frac{1}{2}\left(L_{1}^{\prime}+L_{2}^{\prime}\right)-L_{3}^{\prime}\right| \cdot\left(\frac{\bar{b}}{a}\right) \cdot \sin \phi
\end{gathered}
$$

where $\phi$ is the incident angle of electromagnetic wave, $a_{e}$ is the equivalent radius of dust particles (m), $V_{b}$ is the visibility of dust storms $(\mathrm{Km}), f$ is the frequency of the incident electromagnetic wave $(\mathrm{GHz}) . L_{i}^{\prime}$ and $L_{i}^{\prime \prime}$ [8] are:

$$
\begin{aligned}
& L_{i}^{\prime}=\operatorname{Re}\left[\frac{1}{A_{i}+1 /\left(\varepsilon_{m}^{*}-1\right)}\right] \\
& L_{i}^{\prime \prime}=\operatorname{Im}\left[\frac{1}{A_{i}+1 /\left(\varepsilon_{m}^{*}-1\right)}\right] \quad(i=1,2,3)
\end{aligned}
$$

where $A_{1}=0.243, A_{2}=0.324, A_{3}=0.432$.

The Figure 3 is the relationship of attenuation in the sand-dust storm environment and visibility. $m_{v}\left(\mathrm{~kg} / \mathrm{m}^{3}\right)$ is the water content in the dust, $V_{b 0}$ is the visibility of the dust storms. The curve shows that the higher the visibility is, the smaller the attenuation rate is; with the increase of water content, the attenuation is getting worse.

According to the curve in the Figure 3, it can be seen that in the dry dust storm, the influence that the visibility affects on attenuation is not very clear. However, when the water is in the dust storms, it will seriously affect the attenuation of electromagnetic wave. In the desert hinterland, it can be thought that there is no water in sanddust storm.

\subsubsection{Scattering Influence on Electromagnetic Wave}

When the sand-dust storm happens, there are a large number of dust particles in the air, which can cause the scattering of electromagnetic wave, it reshoots in the attenuation of electromagnetic wave, as well. Although the shapes of dust particles are irregular, the scattering statistics of the dust particle can be equivalent into a sphere particle scattering. So, the analysis of the dust particles can take advantage of the Mie theory [9] for analysis. According to the Mie theory, the scattering and extinction of section are:

$$
\begin{aligned}
& \sigma_{s}=\frac{\lambda^{2}}{2 \pi} \sum_{n=1}^{\infty}(2 n+1)\left(\left|a_{n}\right|^{2}+\left|b_{n}\right|^{2}\right) \\
& \sigma_{t}=\frac{\lambda^{2}}{2 \pi} \sum_{n=1}^{\infty}(2 n+1) \operatorname{Re}\left(a_{n}+b_{n}\right)
\end{aligned}
$$

where $\lambda$ is the wavelength of electromagnetic wave, $a_{n}$ and $b_{n}$ are the scattering coefficient of Mie.

In the sand-dust storm, the distribution of dust particles present in certain scale, the attenuation of carrier frequency caused by unit distance (characteristic attenuation) is:

$$
A=4.343 \times 10^{3} \int_{r_{1}}^{r_{2}} N_{0} \sigma_{t}(r) \rho(r) d r \quad(\mathrm{~dB} / \mathrm{km})
$$

where $\rho(r)$ is the size distribution probability density function dust particles, $N_{0}$ is the density of dust particles per unit volume [10].

Because the measurement of $N_{0}$ is difficult, so in general study, it often uses visibility to describe the concentration of dust particles,

$$
\begin{gathered}
V_{b}=15 / \alpha_{0} \\
\alpha_{0}=8.686 \times 10^{3} \pi N_{0} \int_{r_{1}}^{r_{2}} r^{2} \rho(r) d r
\end{gathered}
$$

where $r_{1}, r_{2}$ are respectively minimum radius and the maximum radius of the dust particles, $\alpha_{0}$ is the attenuation coefficient of electromagnetic wave [11].

According to (22) and (23), dust particles count per unit volume ( $N_{0}$ ) can be calculated:

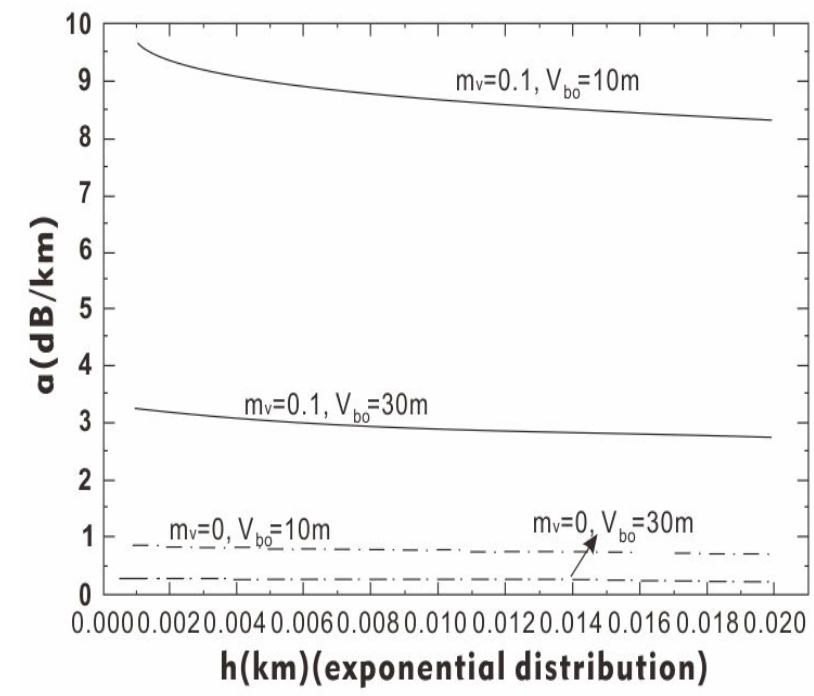

Figure 3. The relationship of attenuation in the sand-dust storm environment and visibility. 


$$
\begin{aligned}
& N_{0}=\frac{15}{8.686 \times 10^{3} \pi V_{b} \int_{r_{1}}^{r_{2}} r^{2} \rho(r) d r} \quad\left(\mathrm{num} / \mathrm{m}^{3}\right) \\
& {\left[\sigma_{s, r}^{2}, \sigma_{r, d}^{2}\right]=[10,1] .}
\end{aligned}
$$

The attenuation of electromagnetic wave scattering characteristics in the sandstorm environment as follows:

$$
A=\frac{15 \int_{0}^{D} \sigma_{t}(r) \rho(r) d r}{2 \pi V_{b} \int_{0}^{D} r^{2} \rho(r) d r}(\mathrm{~dB} / \mathrm{km})
$$

It can be used single dust particles attenuation for the overall calculation [12]. The spread of the electromagnetic wave will be influenced by multiple scattering; it can make use of Markov process. Because electromagnetic wave has the characteristic of light, the sample space after scattering $\mathrm{m}$ times space spread sequence is $\left\{s_{l}\right\}(l=1, \cdots, \mathrm{m})$.

$$
P(s)=\sum_{m=0}^{\infty} P_{m}(s)
$$

$P_{m}(s)$ is the probability that electromagnetic wave propagation in the sandstorm environment after scattering $\mathrm{m}$ times [13]. Because this process can be seen that random Markov process

$$
P(s)=P\left(s_{0}\right) P\left(s_{1} / s_{0}\right) \cdots P\left(s_{m} / s_{m-1}\right)
$$

The conditional probability $P\left(s_{l} / s_{l-1}\right)(l=1, \cdots, m)$ is one probability of electromagnetic wave propagation in space to space after scattering, the estimation function is:

$$
\begin{aligned}
P_{t}= & \sum_{m=0}^{\infty} P_{m}=\sum_{m=0}^{\infty} W_{m} \exp \left[-C_{t} \frac{h-z_{m}}{\cos \alpha_{m}}\right] . \\
& \eta\left(\cos \alpha_{m}\right) \cdot \prod_{l-1}^{m} \eta\left(h-z_{l}\right) \eta\left(z_{l}\right)
\end{aligned}
$$

where $\quad C_{t}=N\left\langle\sigma_{t}\right\rangle$

$$
\eta(x)= \begin{cases}1 & (x>0) \\ 0 & (x \leq 0)\end{cases}
$$

The weight function $W_{m}$ is:

$$
W_{m+1}=W_{m} \exp \left[-C_{\alpha}\left|\frac{z_{m+1}-z_{m}}{\cos \alpha_{m}}\right|\right]
$$

where $\alpha_{m}$ is a angel that scattering direction and the $\mathrm{z}$ axis after electromagnetic wave scattering $\mathrm{m}$ times [14]. The initial weights of Electromagnetic wave is $W_{0}=1$.

$$
C_{\alpha}=N\left\langle\sigma_{\alpha}\right\rangle
$$

The average transmittance of electromagnetic wave is:

$$
T=\frac{1}{N} \sum P_{t}
$$

Under the condition of the atmosphere visibility is higher, the amount of dust particles in the air is small, and the scattering of electromagnetic waves is very weak, scattering need not to be considered. However, when the sand-dust storm is very heavy, the air visibility is less than $1 \mathrm{~km}$, the scattering of dust particles is very strong, it will seriously affect the signal transmission.

\section{Simulation Results and Analysis}

The location of the relay node will affect the performance of the system. Three location of the relay node are analyzed [15].

First: the relay node is located at the source node and destination node precisely symmetric center position. That is

$$
\left[\sigma_{s, r}^{2}, \sigma_{r, d}^{2}\right]=[1,1] .
$$

The second: the location of the relay node is located in the close distance to destination node. That is

$$
\left[\sigma_{s, r}^{2}, \sigma_{r, d}^{2}\right]=[1,10] \text {. }
$$

The third: the location of the relay node is located in the relatively close distance from the source node. That is

$$
\left[\sigma_{s, r}^{2}, \sigma_{r, d}^{2}\right]=[10,1] .
$$

The Figure 4 is the performance curve of relay located in different position. It can be obtained from the figure: a relay node is located in the close distance between source node and destination node position, cooperative communication system performance is better, and in both cases, the performance of system is approximately the same. When the relay nodes in the source node and destination node center position, performance of the system is worse. It is the cause of the equal power allocation.

The Figure 4 is the performance curve in the equal power allocation. The Figure $\mathbf{5}$ is the performance curve in the unequal power allocation. The power allocation is: the source node power is two-thirds of the total power,

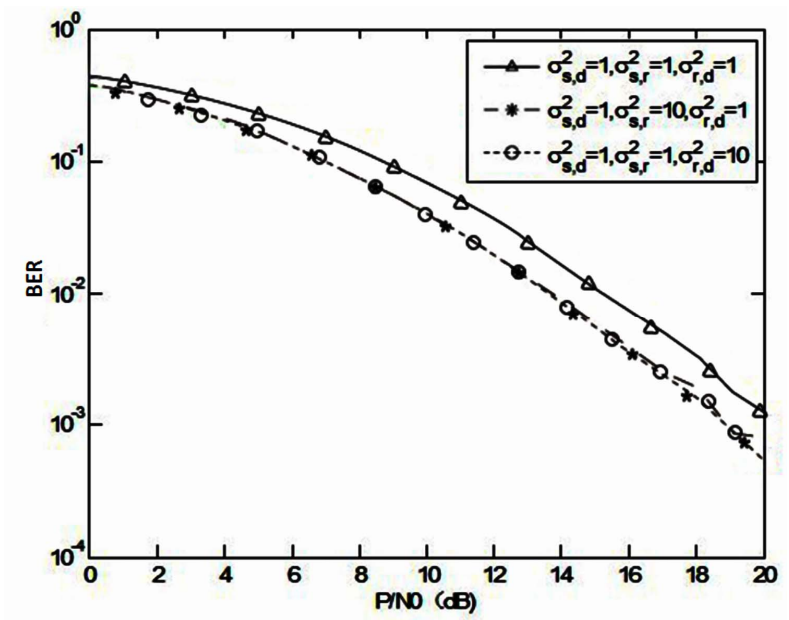

Figure 4. The performance curve of relay located in different position and equal power allocation. 


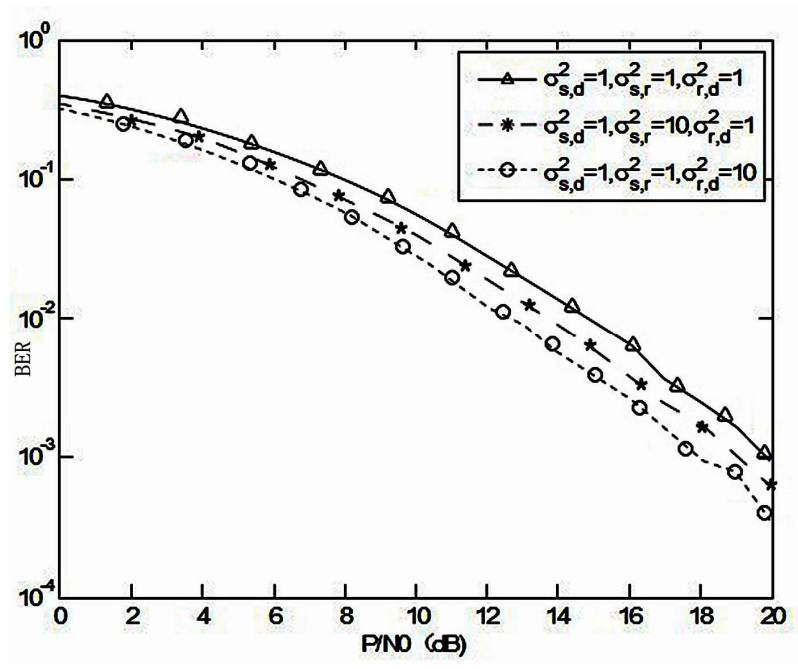

Figure 5. The performance curve of relay located in different position and unequal power allocation.

the relay node power is a third of the total power. It is discussed in accordance with the above three kinds of relay location conditions.

We can obtain from the Figure 5: in ranging from power allocation, the system performance of close distance from the source node is no longer the same as close distance from destination node. Under the condition of the same SNR, close distance relay node to destination node position can bring good system performance. When the relay node is in the close distance to destination node position, collaborative system of communication system performance is best.

\section{Conclusions}

In this paper, we have analyzed two kinds of cooperative communication modes and get the conclusion that the low-order modulation method should be used in cooperative communication. In the analysis of dust environment, polarization and scattering are the two main factors that influence the propagation of electromagnetic wave. The moving of nodes and power allocation will affect the performance of collaborative communication system.

In sand-dust storm environment, the electromagnetic environment is very complex, dust particle scattering of electromagnetic waves become the important factor to influence the signal transmission. When the signal is transmitting, the scattering effect can cause the multipath fading. So the key point of the sand-storm communication is to overcome the influence of multipath fading.

Furthermore, in the future study, OFDM technology can be used in system, because OFDM technology can achieve the role of resistance to multipath fading. OFDM cooperative communication technology can support the quality of communication system.

\section{Acknowledgements}

This work was supported by the Nature Science Foundation of China (61162020). The authors would like to thank the anonymous referees for their valuable comments and suggestions.

\section{REFERENCES}

[1] A. Goldmith, H. W. Yang, W. D. Li and W. B. Guo, "Wireless Communication," People's Posts and Telecommunications Press, 2007.

[2] J. N. Laneman, D. N. C. Tse and G. W. Wornell. “Cooperative Diversity in Wireless Networks: Efficient Protocols and Outage Behavior," IEEE Transactions on Information Theory, Vol. 50, 2004, pp. 3062-3080. doi: 10.1109/TIT.2004.838089

[3] M. K. Simon and M. S. Alouini, "Digital Communication over Generalized Fading Channels: A Unified Approach to Performance Analysis,” IEEE 14th International Conference on Communication Technology, Vol. 86, 1998, No. 9, pp. 1860-1877.

[4] L. Hao, W. Yang and T. W. Wu, "The Optical Properties of Dust Aerosols and Radiation Force Effect," The Chinese desert, Vol. 30, No. 6, 2010, pp. 1477-1782.

[5] N. Huang, X. J. Zheng and G. T. Chen, "Advances on Research of Sand-dust Storm Disturbing Radio Wave's Propagation,” The Chinese desert, Vol. 18, No. 4, 1998, pp. 755-353.

[6] X. M. Wu, "Sandstorm Affect Microwave, Millimeter Wave Propagation," Xi 'an University of Electronic Science and Technology, 1993.

[7] W. Y. Yin and J. M. Xiao, "The Effects of Sand and Dust Storm on Microwave Links," Journal of China Institute od Communications, Vol. 12, No. 51991.

[8] T. S. Chu, "Effects of Sand Storms on Microwave Propagation,” The Bell System Technical, Vol. 58, No. 2, 1979, pp. 549-553.doi:10.1002/j.1538-7305.1979.tb02234.x

[9] R. K. Yang, D.-J. Jian and R.-H. Yao, "Research on Attenuation and the Two-Frequency Mutual Coherence Function for Millimeter Wave Propagation in the Sand and Dust Storm," Journal of Xi 'an University of Electronic Science and Technology (Natural Science Edition), Vol. 12, No. 34, 2007, pp. 953-957.

[10] J. W. Craig, “A New, Simple and Exact Result for Calculating the Probability of Error for Two-dimensional Signal Constellations,” IEEE MILCOM, Boston, MA, Vol. 25, No. 5, 1991, pp. 1-5.

[11] S. I. Ghobrial, "Effect of Hygdroscopic Water on Dielectric Constant of Dust at X-band,” Electronics Letters, Vol. 16, No. 10, 1980, pp. 393-394.doi:10.1049/el:19800277

[12] X. J. Zheng, Z. W. Gao and Y. H. Zhou, "Electromagnetic Wave Monte Carlo Simulation of Propagation in a Sandstorm,”Journal of science in China, Vol. 38, 2008, pp. 955-961.

[13] X. J. Zheng, X. C. Li and L. Xie, “Cross Depolarization Effect of Electromagnetic Wave by Partially Charged 
Spherical Sands in Sandstorms," Journal of Desert Research, Vol. 31, 2011, pp. 567-570.

[14] G. W. Wornell and J. N. Laneman, "Energy-efficient Antenna Sharing and Relaying for Wireless Networks," In Proc. IEEE Wireless Comm. And Net. Conference
(WCNC2000). Chicago, USA, 7-12 September 2000.

[15] F. Elis a. "A New Permittivity Model for the Microwave Moisture Measurement of Wet Sand,” 33rd Europe a Microw Ave Conference-munich, 2003, pp. 539-542. 